\title{
Reconocimiento de los jóvenes desmovilizados de los grupos armados en Colombia: transiciones y desafíos para las prácticas de orientación escolar
}

\section{Resumen}

Este artículo examina cómo los modelos de reconocimiento social contribuyen a las prácticas de orientación escolar de jóvenes desmovilizados de las organizaciones implicadas en el conflicto armado en Colombia, que deciden insertarse al sistema educativo. Los resultados de esta reflexión derivada de investigación se presentan en cuatro apartados. El primero aborda la estructura del reconocimiento social por medio de tres modelos o estadios de reconocimiento intersubjetivo según Georg Wilhelm Friedrich Hegel, Axel Honneth y Paul Ricœur. El segundo comprende las emociones como elementos principales para interpretar las acciones morales de los jóvenes que asumen el reto de reintegrarse a la vida civil. El tercero trata la manera como los jóvenes desmovilizados se constituyen como sujetos que participan en escenarios de socialización ética y política. El cuarto indaga sobre la memoria y la narrativa como condiciones necesarias para que los jóvenes desmovilizados se reconozcan como protagonistas de su propia historia con capacidades de reorientar y reconstruir su vida individual y colectiva.

\section{Palabras clave}

Orientación escolar; reconocimiento; emociones; derechos; memoria; escuela; jóvenes
Escrito por Henry Camilo Bejarano-Sanabria Corporación de Educación Tecnológica Colsubsidio-Airbus Group, CeT BOgOTÁ, COLOMBIA henry.bejarano@colsubsidio.com

Ricardo Mauricio Delgado-Salazar Pontificia Universidad JaVERIANA Bogotá, Colombia rdelgado@javeriana.edu.co

\section{Transferencia a la práctica}

Este artículo de reflexión busca aportar un referente conceptual a las prácticas de orientación escolar, para el diseño y actualización de rutas y protocolos de atención dirigida a jóvenes desmovilizados de los grupos armados ilegales, en el proceso de su vinculación a la educación básica y media.

Para citar este artículo / To cite this article / Pour citer cet article / Para citar este artigo

Bejarano-Sanabria, Henry Camilo \& Delgado-Salazar, Ricardo (2017). Reconocimiento de los jóvenes desmovilizados de los grupos armados en Colombia: transiciones y desafíos para las prácticas de orientación escolar. magis, Revista Internacional de Investigación en Educación, 9 (19), 149-164. doi: 10.11144/Javeriana.m9-19.rjdg 


\section{Keywords}

School orientation; recognition; emotions; rights; memory; school; youth

\section{Mots clés}

Orientation scolaire; reconnaissance; émotions; droits; mémoire; école; jeunes

\section{Abstract}

This article examines how the models of social recognition contribute to the school practices of demobilized young people from the organizations involved in the armed conflict in Colombia, who decide to return to the educationa system. Results of this reflection are presented in four sections. The first section deals with the structure of social recognition through three models or stages of intersubjective recognition according to Georg Wilhelm Friedrich Hegel, Axel Honneth and Paul Ricœur. The second section includes the study of emotions as main elements for interpreting the moral actions of young people who take on the challenge of reintegrating into civilian life. The third section explains how these young people demobilized are constituted as subjects that participate in scenarios of ethical and political socialization. The fourth section investigates memory and narrative, as necessary conditions for demobilized youth, to help them recognize themselves as main actors of their own history, and with the ability to reorient and reconstruct their individual and collective lives.

\section{Transfer to practice}

This article aims to provide a conceptual reference to school practices for the design and updating of routes, and care protocols, of young people demobilized from illegal armed groups in their return to basic and secondary education.

\section{Résumé}

Cet article examine comment les modèles de reconnaissance sociale contribuent aux pratiques d'orientation scolaire des jeunes démobilisés des organisations impliquées dans le conflit armé colombien, qui décident de se réintégrer au système éducatif. Les résultats de réflexion dérivée de la recherche se présentent en quatre parties. La première aborde la structure de la reconnaissance sociale au moyen de trois modèles ou strates de reconnaissance intersubjective selon Georg Wilhelm Friedrich Hegel, Axel Honneth y Paul Ricœur. La deuxième comprend les émotions en tant qu'éléments principaux pour interpréter les actions moraux des jeunes qui prennent le défi de se réintégrer à la vie civile. La troisième s'agit de la manière dont les jeunes démobilisés se constituent en tant que sujets qui participent dans les scenarios de socialisation étique et politique. Enfin, la quatrième partie indague par rapport à la mémoire et à la narrative en tant que conditions nécessaires pour que les jeunes démobilisés se perçoivent en tant que protagonistes de sa propre histoire avec les capacités de réorienter et reconstruire sa vie individuelle et collective.

\section{Transfert à la pratique}

Cet article de réflexion cherche donner un fondement conceptuel aux pratiques d'orientation scolaire, pour le dessin et actualisation de routes et protocoles d'attention dirigées aux jeunes démobilisés des groupes armées illégales, dans les processus d'incorporation à l'éducation secondaire et terminale.

\section{Palavras-chave}

Orientação escoar; reconhecimento; emoções; direitos; memória; escola; jovens

\section{Resumo}

Este artigo examina como os modelos de reconhecimento social contribuem às práticas de orientação escolar de jovens desmobilizados das organizações implicadas no conflito armado na Colômbia, que decidem inserir-se no sistema educativo. Os resultados desta reflexão derivada de pesquisa apresentam-se em quatro partes. A primeira aborda a estrutura do reconhecimento social por meio de três modelos ou estágios de reconhecimento intersubjetivo segundo Georg Wilhelm Friedrich Hegel, Axel Honneth e Paul Ricœur. O segundo compreende as emoções como elementos principais para interpretar as ações morais dos jovens que assumem o desafio de reintegrar-se à vida civil. O terceiro trata da maneira como os jovens desmobilizados se constituem como sujeitos que participam em cenários de socialização ética e política. O quarto indaga sobre a memória e a narrativa como condições necessárias para que os jovens desmobilizados se reconheçam como protagonistas de sua própria história com capacidades de reorientar e reconstruir sua vida individual e coletiva.

\section{Transferência à prática}

Este artigo de reflexão procura oferecer um referente conceitual às práticas de orientação escolar, em vista do desenho e da atualização de rotas e protocolos de atenção dirigidos a jovens desmobilizados dos grupos armados ilegais, no processo de sua vinculação à educação básica e média. 


\section{Introducción}

Este artículo de reflexión es uno de los productos académicos originados de la investigación Prácticas de reconocimiento y de orientación dirigidas a niños desvinculados y jóvenes desmovilizados de los grupos armados ilegales, en la educación básica y media. Estudios de caso en instituciones educativas de la ciudad de Bogotá, adelantada en el contexto del grupo de investigación Educación para el Conocimiento Social y Político, de la Facultad de Educación de la Pontificia Universidad Javeriana de Bogotá, entre 2014 y 2015. El propósito central de la investigación se orientó a comprender desde las prácticas de orientación escolar que se brindan en algunas de las instituciones educativas distritales que acogen a esta población, las dificultades y oportunidades que se presentan en los procesos de orientación y acompañamiento de los jóvenes desmovilizados. El proyecto estableció una metodología mixta que contempló por una parte, la revisión documental para la elaboración del estado del arte que incluye algunas aproximaciones investigativas y conceptuales en torno a los procesos de reconocimiento y vinculación al sistema educativo de los jóvenes que estuvieron incorporados en los grupos alzados en armas. Y por otro lado, se consideró un diseño metodológico basado en el enfoque narrativo como estrategia de investigación que pretendió focalizar y resaltar la voz de los orientadores escolares de las instituciones educativas de las localidades de Ciudad Bolívar, Usme y Kennedy, mediante técnicas de recolección de información como la entrevista semiestructurada y el grupo focal.

Por consiguiente, el presente artículo de reflexión — derivado de los resultados de esta investigación - busca por una parte profundizar en la comprensión de las dimensiones asociadas al reconocimiento de los jóvenes desmovilizados del conflicto armado a partir de los testimonios de los orientadores escolares que han asumido las prácticas de acogida de esta población en el contexto escolar. Y por otra parte, aportar a los orientadores escolares un referente conceptual que les permita cualificar y sustentar sus prácticas de orientación.

\section{Escuchar la voz de los orientadores escolares}

El orientador escolar es una figura imprescindible en la escuela para los procesos de atención de los jóvenes que decidieron desmovilizarse de los grupos armados ilegales. Los resultados de las narrativas de los orientadores señalan aspectos relacionados con las dimensiones del re-

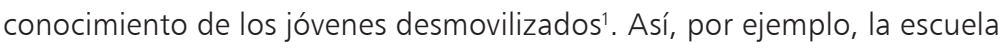
en su proyecto de constituirse como escenario de acogida y protección requiere proporcionarle al desmovilizado el reconocimiento social de su dignidad como persona. Así lo describió una orientadora sobre el proceso de atención de un joven desmovilizado: "siempre sintieron acá que los tratamos como personas y que siempre tuvieron aquí a alguien con quién contar y ese me parece que fue el éxito que nosotros tuvimos acá" (E). Sin embargo, los orientadores reconocen la falta de cualificación para orientar los procesos de acogida de los jóvenes desmovilizados: "esa es la preocupación que le surge a uno como orientador: ¿qué va a venir después de que se haga la paz? Porque es que es a nosotros los educadores es a quienes nos han puesto ese rol, de atender a esta población sin
Descripción del artículo | Article description | Description de l'article | Artigo descrição

En este artículo de reflexión, derivado del proyecto Prácticas de reconocimiento y de orientación dirigidas a niños desvinculados y jóvenes desmovilizados de los grupos armados ilegales, en la educación básica y media. Estudios de caso en instituciones educativas de la ciudad de Bogotá, los investigadores abordan desde la Filosofía moral y política, las implicaciones afectivas, jurídicas y sociales que tiene el reconocimiento de los jóvenes desmovilizados de los grupos armados en Colombia; y los desafíos que esto representa para la escuela, en el proceso de vinculación e inclusión de esta población al sistema educativo.

1 Las narraciones derivadas de las entrevistas que resaltan la voz y el pensar de los orientadores escolares serán caracterizadas con la nominación E. Magis no edita las transcripciones de las entrevistas y respeta las marcas orales de los entrevistados. 
herramientas, sin apoyo, ni motivación. ¿Sí? Nos ha tocado, prácticamente con el sentido común y con la experiencia que tenemos simplemente de manejo psicosocial" (E).

Del mismo modo, algunos resultados de la investigación nos permiten comprender que la escuela requiere ser pensada como un lugar para el desarrollo del reconocimiento mutuo. A los jóvenes desmovilizados "los reconocí como personas, me interesó realmente lo que les pasaba, y ellos lo podían percibir. Nunca tomé un lápiz. Nunca tomé una hoja. Y me parece que ese fue el gran éxito. Nos sentábamos como dos amigos a charlar. Y por eso fue que él abrió la puerta" (E). Reconocer y resguardar la dignidad del sujeto desmovilizado es importante para la reivindicación de sus derechos. En efecto, en el escenario normativo del reconocimiento, toda persona es tenida en cuenta como portadora de derechos cuando se le reconoce como actor social. De este modo, "es necesario trabajar mucho desde la parte del enfoque de derechos. La restitución de derechos es una obligación de todos y todos debemos ser garantes de sus derechos", decía una orientadora (E). Además, los desmovilizados "se convierten en líderes positivos y se dan a querer muchísimo porque tienen la calma y la tranquilidad que de pronto no tienen los otros chicos" (E).

De hecho, una de las orientadoras señaló la importancia de restituirlos como "sujetos constructores de paz: iNunca! se habla de víctimas, sino de niños constructores de paz, ellos están haciendo un trabajo dentro de la institución que es muy importante, porque generan actividades de convivencia y de paz en el colegio" (E). En palabras de una orientadora: "muchas veces he tenido la oportunidad de ver cómo ellos mismos les dan consejos a otros, más pequeños de que venga, no haga esto, no se meta con estas personas, así que ellos empiezan a ver que depende mucho de ellos, para que empecemos a cambiar esos estereotipos y más esas etiquetas" (E).

Sin embargo, no podemos perder de vista que el reconocimiento que se da en el diario vivir, mediante las interacciones con los demás, necesita constituirse en elemento social que identifique en la población desmovilizada jóvenes capaces de reorientar y reconstruir su vida individual y colectiva en clave ética y política. Es cierto que algunos jóvenes que han vivido crueles experiencias de guerra anhelan regresar con sus familias, reorientar sus proyectos de vida o recuperar las vidas que dejaron atrás. En efecto, "ellos traen la decisión clara de que quieren cambiar de vida, que desertaron, buscaron la manera de salir ya de esa vida y dejarla atrás, porque encontraron que no tenía un significado para ellos y de pronto añoran encontrarle un nuevo sentido a su vida, entonces ellos llegan con la intención y eso es el apoyo que les hacemos de resignificar nuevamente, todo lo que han vivido y de reconstruir su proyecto de vida (...) a partir de la historia de vida, se pregunta dónde estoy ahora, tejiendo sueños construyo un proyecto y un sueño para mi historia de vida y se plantea otro, es decir, la reconstrucción" (E).

A partir de los anteriores aspectos, consideramos que el reconocimiento es, sin duda, un referente que se aplica en la comprensión de los procesos humanos de socialización, la intersubjetividad, el proceso de formación ética de los sujetos y el sistema normativo de la sociedad. Además, los procesos de reconocimiento cumplen una función generadora de dedicación emocional, normativa y social para la atención a jóvenes desmovilizados del conflicto armado en Colombia en el contexto escolar. Desde esta perspectiva, la escuela requiere ser un espacio que promueva los diferentes modos de sociabilidad humana del amor, el derecho y la solidaridad (Honneth, 1997, 2010). Es decir, la escuela debe involucrarse en dinámicas sociales que susciten prácticas de acogida y protección a la población que 
ha sufrido directamente la dimensión y la complejidad de la violencia, en especial los jóvenes desmovilizados. Por eso, desde las políticas públicas y las prácticas pedagógicas, es importante generar acciones encaminadas a que la escuela con la ayuda de los orientadores escolares se convierta y se reconozca como un territorio de paz, donde los jóvenes desmovilizados del conflicto armado aprendan a elaborar proyectos para elegir alternativas de vida; logren reivindicar sus derechos; propicien progresivamente una posición positiva frente a sí mismos mediante la autoconfianza, el autorrespeto y la autoestima; fortalezcan sus capacidades y cualidades; y, finalmente, asuman sus deberes y responsabilidades por medio de experiencias que los constituyan como sujetos éticos y políticos.

Los relatos de las entrevistas de los orientadores escolares con experiencia en el acompañamiento de la población desmovilizada se constituyeron en la fuente para la construcción del presente artículo. Desde esta perspectiva, la tesis que se quiere proponer en este escrito plantea que la escuela en su proyecto de constituirse en territorio de paz en un escenario de posconflicto armado, requiere ser un lugar que promueva prácticas generadoras de reconocimiento afectivo, jurídico y social que aporten a los jóvenes que participaron en los grupos alzados en armas, oportunidades y caminos que les permitan reorientar y reconstruir sus itinerarios de vida con dignidad y justicia. Así, el referente teórico desde el cual se fundamentó esta reflexión tiene como base la filosofía política y la filosofía moral, en respuesta, precisamente, a las necesidades de aportar a la formación de los orientadores escolares un referente conceptual que les permita sustentar sus prácticas de reconocimiento a la población desmovilizada. En este sentido, se acudió a la investigación documental consultando el trabajo intelectual sobre las teorías del reconocimiento presente en Georg Wilhelm Friedrich Hegel $(1973,2006)$, Axel Honneth $(1997,2009,2010)$ y Paul Ricœur (2001, 2003, 2004, 2006); el ejercicio teórico sobre las emociones, desarrollado por Martha C. Nussbaum (2008); y la reflexión de Manuel Reyes Mate $(2003,2006)$ sobre la memoria y la justicia.

El artículo se ha estructurado en cuatro apartados: el primer aspecto de este escrito se centra en la descripción de la categoría de reconocimiento. Este segmento, Formas de socialización. La comprensión de las relaciones del reconocimiento social, considera la propuesta de Axel Honneth $(1997,2009,2010)$ respecto a la lectura progresiva del concepto de reconocimiento en Hegel. Se retoman igualmente los aportes de P. Ricœur (2006), quien desde su libro Caminos del reconocimiento, desarrolla tres estudios del reconocimiento —el reconocimiento como identificación, el reconocimiento de sí mismo y el reconocimiento mutuo- a partir de la "polisemia regulada" del verbo "reconocer".

El segundo apartado que se presenta trata sobre la dimensión emocional de los jóvenes desmovilizados en el proceso de la orientación escolar, y aborda la relevancia de las emociones; teniendo como referencia los planteamientos de Martha C. Nussbaum, (2008) en su libro Paisajes del pensamiento, quien destaca las emociones como generadoras especialmente poderosas para fomentar condiciones del bienestar en una cultura política.

El tercer apartado, El reconocimiento de jóvenes desmovilizados de los grupos armados en Colombia como sujetos ético-políticos, comprende la relación jurídico-normativa de los procesos intersubjetivos en virtud de la cual los jóvenes que participaron en experiencias de guerra se consideran sujetos de una relación recíproca entre personas de derechos, capacidades y deberes. El cuarto apartado, cuyo tema es sobre la memoria y la justicia de los jóvenes desmovilizados en el proceso de la orientación 
escolar, describe el papel de la justicia y la memoria que subyace en la existencia de estos jóvenes, teniendo como referencia los argumentos aportados por Manuel Reyes Mate y Paul Ricœur.

\section{Resultados}

Formas de socialización. La comprensión de las relaciones

del reconocimiento social

El concepto de reconocimiento (Anerkennung) de Georg Wilhelm Friedrich Hegel es un referente que se aplica en la comprensión de los procesos humanos de socialización, la intersubjetividad, el proceso de formación ética de los sujetos y la estructura normativa de la sociedad. Hegel recoge la teoría del reconocimiento de Johann G. Fichte para describir el marco normativo de las formas de relaciones éticas en sus escritos de Jena. En su escrito sobre el Fundamento del derecho natural, Fichte había conceptualizado el reconocimiento como una interacción entre individuos que basan sus modos de socialización en un modelo de relaciones jurídicas (Honneth, 1997). El modelo filosófico que Hegel elaboró en Jena tenía como propósito explicar el proceso de formación ética del espíritu humano por medio de tres formas elementales de socialización: la familia, el derecho y la comunidad ética. Axel Honneth observa esta concepción al comienzo de su obra consagrada a la lucha por el reconocimiento.

De la reconstrucción previa de los escritos de Hegel (2006) en Jena, esto es, de El sistema de eticidad, Axel Honneth (1997) toma la idea del encadenamiento de "tres modelos de reconocimiento intersubjetivo"2, colocados, sucesivamente, bajo el nombre del amor, del derecho y de la estima social. En los tres modos prima el punto de la intersubjetividad y la comunicación y conservan de Hegel el proyecto de crear una teoría social normativa. Honneth (1997) describe que durante largo tiempo de su vida, Hegel en su filosofía política tenía la convicción de que "la lucha de los sujetos por el recíproco reconocimiento de su identidad comportaba una necesidad social interna de la aceptación de instituciones que garantizasen la libertad" (p. 13). De esta manera, Hegel entiende el modelo de reconocimiento como un proceso de relación intersubjetiva que se constituye en una interacción entre individuos. Sin embargo, toda interacción que establezca presupuestos intersubjetivos necesita interpretarse desde la perspectiva particular o subjetiva de cada individuo. De este modo,

2 Axel Honneth (1997) realiza una separación dependiente de las tres formas de reconocimiento: amor (familia), derecho (Estado), valoración (sociedad civil). Honneth justifica las relaciones de reconocimiento social, a partir de la esfera del amor, del derecho y la solidaridad. Para Honneth (1997), el amor — lazos afectivos - representa el primer estadio de reconocimiento recíproco. En esa medida, esta relación de reconocimiento está ligada a la existencia corporal del otro concreto y los sentimientos de uno al otro proporcionan una valoración específica. La esfera del amor se configura como un modo singular, donde la exigencia del reconocimiento solo se comprende en la interacción íntima de los sujetos. Esto hace — según Honneth — que las realizaciones morales del cuidado del sujeto residan sobre una base afectiva. La esfera del derecho comprende la formación moral del reconocimiento recíproco. Según Honneth (1997), Hegel había señalado el hecho de que "no podemos llegar al entendimiento de nosotros mismos como portadores de derechos, si no poseemos un saber acerca de qué obligaciones normativas tenemos que cumplir frente a los otros ocasionales" (p. 133). Según esta concepción, "vivir sin derechos individuales significa, para el miembro de la sociedad, no tener ninguna oportunidad para la formación de su propia autoestima" (p. 133). La última de las esferas llama la atención sobre el proceso de formación ética de la especie humana, el cual se alcanza a partir de la existencia de compromisos solidarios intersubjetivos como condición de todo proceso humano de socialización. Además, "la pregunta por el estado de la estructura del reconocimiento de una sociedad determinada no puede contestarse sino con estudios que analicen el estado empírico que guardan las concreciones institucionales de cada uno de estos tres patrones de reconocimiento" (Honneth, 2009, p. 267). 
es importante que la escuela promueva la configuración de la subjetividad y la afirmación de sí que desea alcanzar cada sujeto. Para esto, es preciso abrir espacios donde la socialización y el respeto por la pluralidad del otro en el diario vivir permitan al sujeto afirmar su diferencia en el reconocimiento del otro.

En el marco del esquema tripartito de las esferas o estadios de socialización humana, inspirados por los escritos de Hegel en Jena, Honneth decidió subrayar en la tercera etapa de su "actualización sistemática", el concepto de estima social o solidaridad. Tanto Hegel como Honneth han diferenciado respecto del amor y de la relación jurídica, una más amplia forma de reconocimiento que solo puede concebirse adecuadamente si como presupuesto se piensa la existencia de un horizonte de valores compartidos. Por este motivo, la solidaridad — como forma de sociabilidad - contiene todas las modalidades del reconocimiento que excede el primer modelo expuesto con el nombre del amor, en el que "los sujetos recíprocamente se confirman en su naturaleza necesitada y se reconocen como entes de necesidad" (Honneth, 1997, p. 118) en un plano de relaciones eróticas, de amistad o familiares. Del mismo modo, sobrepasa el segundo modelo comprendido en el plano normativo de los derechos, en el que los sujetos son aceptados como miembros de una comunidad y las relaciones jurídicas establecen desde un reconocimiento mutuo, la valoración o aprecio social como una forma de reconocimiento intersubjetivo. Igualmente, Paul Ricœur (2006) desarrolla tres estudios del reconocimiento —el reconocimiento como identificación, el reconocimiento de sí mismo, y el reconocimiento mutuo-, a partir de la "polisemia regulada" del verbo "reconocer". Esto es la graduación que va del reconocimiento como identificación de algo, que pasa por el reconocimiento de uno mismo, para llegar al reconocimiento mutuo.

Promover las prácticas generadoras de reconocimiento por parte de los orientadores en los procesos educativos conlleva una explicación conceptual compleja y amplia por desarrollar. Según Ricœur (2006), la dimensión social del reconocimiento comprende la progresión de la temática de la identidad, luego la de la alteridad $y$, finalmente, la de la dialéctica entre reconocimiento y desconocimiento. Esto es sobre cómo el hombre actuante y sufriente, hasta llegar al reconocimiento de lo que él es en verdad, un hombre capaz, exige en cada etapa la ayuda del otro. Según lo dicho, en primer lugar, se requiere tener en cuenta la capacidad que posee el joven de hacer que algo ocurra, la capacidad que posee de comenzar algo por sí mismo, la capacidad que posee de reorientar su vida para mejorar. En segundo lugar, se necesita tener presente en la vida de los jóvenes desmovilizados la "fenomenología del hombre capaz" (Ricœur, 2003, p. 121). Desde esta concepción, el sujeto en el redescubrimiento de su identidad personal es capaz de poder contar y contarse. En tercer lugar, implica sustituir el "estado de lucha", bajo el cual se ha articulado la reflexión tradicional sobre el concepto (a partir del argumento hegeliano), por un "estado de paz"3.

En la perspectiva de la identidad, la alteridad y el estado de paz, la educación debe ser un espacio donde los sujetos singulares se encuentren. Un lugar donde "el sujeto humano le debe su identidad a la experiencia de un reconocimiento intersubjetivo" (Honneth, 1997, p. 90). En este caso, la alteridad requiere un valor importante en la relevancia social y con ella la exigencia de que los otros sean aceptados desde sus vidas diferenciables. A este respecto, una de las orientadoras señaló cómo la escuela se convierte en un espacio de reconocimiento y de inclusión de los jóvenes desmovilizados: "frente a los compañeros el reconocimiento está marcado por la aceptación, no lo ven como el diferente, creo que esto es una fortaleza, y es algo muy bonito de resaltar por lo menos dentro de mi experiencia" (E).

Sin embargo, de las tres formas de reconocimiento intersubjetivo, la comprensión de las emociones requiere proporcionar claramente la base inicial, sobre la cual los jóvenes desmovilizados, que asumieron experiencias de menosprecio, indignación, culpa, compasión, pueden restituirse como sujetos ético-políticos y mejorar el entendimiento positivo de sí mismos paralelamente con los procesos de "autorrespeto", "autoconfianza" y "autoestima". Por esta razón, acogiendo los planteamientos de Martha $C$. Nussbaum (2008), la escuela con ayuda de los orientadores escolares necesita ofrecer espacios donde las emociones fomenten las condiciones de bienestar emocional de una cultura política.

Reflexiones en torno a la dimensión emocional de los jóvenes desmovilizados en los procesos de la orientación escolar

El segundo aspecto de este texto señala la importancia de las emociones en los procesos de orientación escolar de los jóvenes desmovilizados. La escuela y en ella los orientadores escolares están llamados a discernir acerca de las emociones de los jóvenes que participaron en experiencias de guerra. Cabe anotar que los orientadores requieren comprender que algunos jóvenes desmovilizados presentan un tipo de

3 Desde esta perspectiva, Ricoeur (2006) hace una apuesta a favor del reconocimiento, desde los modelos de estado de paz conocidos por su denominación griega de origen, esto es, "la philia Aristótelica, el eros Platónico, y el agape bíblico" (p.277). Los modelos de estado de la philia, el eros, el agape, Ricoeur los compilará ampliamente en su texto Amor y justicia. En este texto, Ricoeur (2001) propone pensar en "la dialéctica entre amor y justicia buscando la mediación frágil entre los dos extremos" (p. 15). 
actitudes y sentimientos reactivos "personales" y además crisis en relación con la comprensión de su mismidad. En especial, la crisis resultado de la afectación del daño ocasionado da a lugar a la pérdida de confianza, en relación con el papel que ocupa en la sociedad. Así, emociones como la ira, el desprecio, la indignación, la vergüenza y la culpa, por ejemplo, se desencadenan en respuesta a la ruptura de ciertos códigos morales o a la vulneración de sus derechos fundamentales. En algunos casos, también podemos encontrar los sentimientos de enojo e indignación que vivencian algunos desmovilizados consigo mismos, con los otros y con el mundo, los cuales muestran sucesos de ruptura con sus itinerarios de vida, con su familia y la sociedad. Desde esta concepción, una práctica generadora de reconocimiento en la escuela necesita basarse en la comprensión de las emociones ${ }^{4}$ que develan los sujetos desde su experiencia, antes, durante y después en relación con el conflicto armado.

Es ciertamente claro que en las dinámicas violentas del conflicto armado aparece el peligro de una lesión moral que puede sacudir la identidad de su persona en su totalidad. Esto, que a veces es percibido como una lesión moral, está sometido a diversas formas de menosprecio. Estas formas de menosprecio (maltrato corporal, desposesión de derechos, indignación) particularmente asumidas por jóvenes que participaron en experiencias de guerra van acompañadas de sentimientos de vergüenza, culpa, indignación o tristeza. Sin embargo, frente a las experiencias de menosprecio se reconocen tres distintas esferas de reconocimiento allí donde la forma de la subjetividad de las personas ha sido lesionada. Estas esferas, según Honneth (1997, 2009, 2010), son la esfera del amor, la esfera del derecho y la esfera de la valoración social. A cada una de estas esferas le corresponde un tipo de

4 Martha C. Nussbaum (2008) menciona también la importancia de las emociones y la primera infancia. Desde esta perspectiva, las emociones de un ser humano adulto no pueden entenderse si no se comprende la historia de su primera infancia y niñez. Esto tiene qué ver con que todas las emociones humanas se refieren, al menos en parte, al pasado y llevan consigo las trazas de una historia que es, a un mismo tiempo, idiosincrasia común para todos los humanos y construida socialmente. Las primeras emociones se desarrollan gradualmente, a medida que el pequeño se va haciendo más y más consciente de la importancia que tales transformaciones revisten para su vida, así como del hecho de que, por decirlo de algún modo, llegan desde afuera. Así pues, el primer modelo de reconocimiento de Honneth (1997) expuesto con el nombre del amor abarca la gama de las relaciones eróticas, de amistad o familiares. Se trata, en este caso, de un reconocimiento en el que los sujetos "se confirman en su naturaleza necesitada y se reconocen como entes de necesidad" (p. 118). Es probable que los jóvenes que participaron del conflicto en la experiencia recíproca de atención amorosa en cierto modo como entes de necesidad hayan sido directamente satisfechos o rechazados desde su pasado. Siendo así, es probable que sus emociones más antiguas sean el miedo y la angustia. daño o formas de menosprecio: maltrato y violación a la integridad física en la esfera del amor; desposesión de derechos y exclusión en la esfera del derecho; e indignación e injuria en la esfera de la valoración social.

En la primera esfera, el reconocimiento que se da en contraposición a la humillación física es el amor. A partir del amor se forma la autoconfianza elemental de una persona. Solo a partir de este tipo de reconocimiento se podrán originar los demás modos del reconocimiento. En la segunda esfera, el reconocimiento que se origina en oposición a la exclusión es el derecho. En esta forma de reconocimiento, el individuo se considera, desde los otros participantes en esta interacción, poseedor de derechos. Así, cuando se comprende la relación de derecho como una forma de reconocimiento recíproco se forma el autorrespeto de una persona. En la tercera esfera, el reconocimiento que responde a la forma de menosprecio de la indignación e injuria es la valoración social. En este sentido, cuando los sujetos obtienen la apreciación social de las capacidades que han adquirido a lo largo de la vida se forma la autoestima de una persona. Pues bien, estas esferas en la medida en que se establezcan en las prácticas de orientación escolar, promoverán en los jóvenes desmovilizados "una autorrealización práctica" o un "sentimiento del propio valor" (Honneht, 2010) en los cuales se les puedan garantizar su dignidad o integridad 5 .

Ahora bien, en lo que respecta a la dimensión afectiva, los orientadores escolares requieren tener presente que las emociones no se conciben únicamente como "movimientos irreflexivos". Así, por ejemplo, para Martha C. Nussbaum (2008), las emociones son elementos esenciales de la inteligencia humana, y no meros apoyos o puntales de la inteligencia. Pues bien, la defensa de una teoría cognitiva-evaluadora de las emociones contiene tres ideas relevantes que, según Nussbaum (2008), son la base para diferenciar las emociones de los impulsos naturales: a) la idea de una valoración cognitiva o evaluación, b) la idea del florecimiento propio o de los propios objetivos y proyectos importantes, y c) la idea de la relevancia de los objetos externos como elementos en el esquema de los propios objetivos.

5 En Reconocimiento y menosprecio (2010), Honneth explica una idea de "vida satisfactoria" y de "autorrealización" a partir de formas de socialización del amor, el derecho y la solidaridad: "parece, pues, que con estos tres modelos de reconocimiento - los del amor, el derecho y la solidaridad - quedan establecidas las condiciones formales de relaciones de interacción en el marco de las cuales los humanos pueden ver garantizadas su dignidad o su integridad. Integridad significa aquí, simplemente, que el individuo puede sentirse apoyado por la sociedad en todo el espectro de sus autorrelaciones prácticas" (p. 30). 
Las emociones tienen un papel esencial en el reconocimiento de los jóvenes. Así, los agentes de la escuela requieren considerar que el contenido de las emociones está vinculado a las formas en que los sujetos conciben el mundo, es decir, sobre cómo los jóvenes tienen la capacidad de darle un valor fundamental a la idea de reconstruir sus trayectorias de vida. Desde este enfoque, la concepción cognitiva-evaluadora e intencional de las emociones implica que en sí integre la búsqueda del florecimiento por parte de una persona. Para Martha C. Nussbaum, el hecho de que los seres humanos deliberen éticamente sobre cómo vivir supone que las emociones son una parte fundamental de la deliberación moral. La escuela con ayuda de los orientadores escolares está llamada a ofrecer espacios para que los jóvenes reorienten sus emociones en razón de "dirigirlas hacia" una serie de acciones que promuevan la gratitud, la devoción y la admiración por parte de todos los miembros de una sociedad. Para Honneth (1997), el fundamento de esto ha de verse en la dependencia del "hombre respecto de la experiencia del reconocimiento: para llegar a una autorregulación lograda, el hombre se encuentra destinado al reconocimiento intersubjetivo de sus capacidades y operaciones" (p. 165).

Conviene subrayar que las emociones superan el escenario de la creencia, la intención y la asignación de valor de este objeto. Y esto es así, porque las emociones están estrechamente conectadas con la acción. Martha C. Nussbaum (2008) menciona que las emociones nos conducen hacia un componente significativo de nuestro bienestar y registran la manera en que las cosas se hallan respecto de ese componente importante. A veces, en combinación con las percepciones y las creencias relativas a la situación que están inmediatamente disponibles, este reconocimiento valorativo originará directamente motivaciones para actuar. Desde esta perspectiva, los jóvenes requieren orientar sus emociones hacia metas que estén imbuidas de algún tipo de valor o importancia que se vinculen al esquema sobre la realización de una vida buena "con y para el otro en instituciones de justicia" (Ricœur, 2003, p. 176).

En lo que respecta a emociones como la compasión ${ }^{6}$, la escuela debe formar en una educación moral ${ }^{7}$ que propicie el reconocimiento del otro en una comprensión que no se base en la revictimización del sujeto en cuestión, sino desde la generación de emociones como la admiración, la empatía y la compasión. Hay emociones que se desencadenan por la percepción del sufrimiento del otro, como la compasión. Martha C. Nussbaum (2008) señala que la forma de comprender la estructura de la compasión es pensar en la estructura del yo y de sus preocupaciones. Desde la concepción de las emociones, la escuela está llamada a orientar procesos formativos que desarrollen una racionalidad ético-compasiva que despliegue la comprensión real

6 En ocasiones, la compasión se confunde en algunos sentidos con la empatía. Nussbaum (2008) menciona que esta última no es más que una reconstrucción imaginativa de la experiencia de otra persona, ya sea que la experiencia sea triste o feliz, placentera o dolorosa. El análisis que hace la filósofa a partir de la concepción cognitiva de la compasión es amplio. Por ejemplo, "el primer requisito cognitivo de la compasión es una creencia o una evaluación según la cual el sufrimiento es grave, no trivial. El segundo es la creencia de que la persona no merece ese sufrimiento. El tercero es la creencia según la cual las posibilidades de las personas que experimentan la emoción son parecidas a las del que padece el sufrimiento. En este caso, así como las otras emociones importantes, la compasión está ligada al valor; de ahí que para esta pensadora por encima del amor y la aflicción, la compasión empuja los límites del yo más lejos de lo que se puede pensar" (p. 345).

7 En su libro Las fronteras de la justicia, Nussbaum (2007) señala que Jean Jacques Rousseau dedica una atención sostenida a la maleabilidad de los sentimientos morales, a la posibilidad de cultivarlos por medio de la educación. En este sentido, Rousseau en su Emilio "atribuye buena parte de las injusticias a una educación sentimental perversa y propone una educación —basada en la compasión - que favorezca la justicia social" (p. 403). 
del sufrimiento humano. Para Nussbaum, las emociones tienen componentes cognitivos indispensables para interpretar ciertas situaciones en el plano ético, político y jurídico.

Puesto que las emociones tienen un sentido cognitivo y son educables, subrayamos la importancia de una educación que promueva la práctica de la compasión. La compasión resulta ser una emoción benéfica para la vida pública pues permite tomar en serio las pérdidas ajenas y comprender el dolor de un país que ha sido protagonista de acciones de violencia. La escuela está orientada a cultivar en todos los niveles la capacidad de imaginar las experiencias de otros y de participar en sus sufrimientos. Además, la educación está llamada a darles un amplio espacio a las humanidades y a las artes, desde la escuela primaria en adelante, a medida que los niños van aprendiendo más y más juicios correctos y se van haciendo capaces de ampliar su empatía a más personas y a más diversos estilos de vida (Nussbaum, 2008). Por eso, "en el colegio tendría que haber más oportunidades de maestros de artes y música" (E), como instrumentos didácticos que permitan que los jóvenes "se enamoren del colegio", decía una orientadora. En este aspecto, Martha C. Nussbaum (2010) señala que si las capacidades de las personas en la escuela están asociadas al tema de las humanidades y las artes nuestras relaciones estarán basadas en el respeto y en la comprensión del otro como ser humano, y no como objeto.

Como se advierte, las emociones como componentes cognitivos indispensables para interpretar ciertas situaciones en el plano ético, necesitan ser tenidas en cuenta en la educación, en las instituciones políticas y en la sociedad en general. De este modo, la concepción acerca de formar en las emociones morales está determinada en la idea de una racionalidad ética y emocional. Los sujetos, tanto los que están en la escuela como los jóvenes que serán incluidos en los procesos educativos, necesitan fundar sus creencias y actitudes acerca del proyecto de consolidar una cultura de paz superando sus juicios negativos mediante un reconocimiento mutuo que los legitime como sujetos éticos y políticos. De la misma manera, los agentes que participan en la escuela requieren construir una intencionalidad ética que propicie una vida buena para todos a partir del cultivo de las emociones.

No obstante, la esfera de las emociones no es suficiente para comprender cómo el desmovilizado en una relación de reconocimiento recíproco se considera como portador de derechos cuando se le reconoce como actor social. En este caso, el joven desmovilizado no puede llegar al entendimiento de sí mismo como portador de derechos, si no posee un saber acerca de qué obligaciones normativas requiere cumplir frente a los otros. Así pues, conocer el concepto de "reconocimiento jurídico" (Honneth, 1997), la "intencionalidad ética" (Ricœur, 2003) y la vida buena en el contexto de los jóvenes desmovilizados implicaría pensar en la oportunidad que tienen de ser nuevamente miembros de una sociedad y de establecer pretensiones socialmente aceptadas.

El reconocimiento de jóvenes desmovilizados de los grupos armados en Colombia como sujetos ético-políticos.

Una reconstrucción social

El tercer aspecto de este escrito comprende la relación normativa de los procesos intersubjetivos, en virtud de la cual los jóvenes desmovilizados se consideran sujetos de una relación recíproca entre personas de derechos, capacidades y deberes. En este apartado, la educación se convierte en espacio para la socialización humana en clave de inclusión y de justicia. Esto nos lleva a observar la esfera jurídica ${ }^{8}$ que plantea Honneth (1997), la cual comprende la formación moral del reconocimiento recíproco.

El reconocimiento jurídico debe comprenderse como aquella relación en la que las personas se respetan recíprocamente como sujetos de derecho. En este sentido, un joven que vive en la experiencia del reconocimiento jurídico se reconoce como portador de pretensiones legítimas y como actor moralmente responsable. Además, una persona que comparte con todos los miembros de la comunidad las facultades que la hacen capaz de participar en la construcción de una sociedad democrática constituirá en su formación el fortalecimiento del respeto de sí. Y la posibilidad de referirse a sí mismo positivamente en tal forma, es lo que Honneth (1997) denomina autorrespeto; esto lleva a pensar que "vivir sin derechos individuales significa, para el miembro de la sociedad, no tener ninguna oportunidad para la formación de su propia autoestima" (p. 147). Para Paul Ricœur (2006), "esta estructura del reconocimiento jurídico consiste, pues, en la conexión entre la ampliación de la esfera de los derechos reconocidos a las personas y el enriquecimiento de las capacidades que estos sujetos se reconocen" (p. 250).

8 Comprender el concepto de reconocimiento jurídico en el contexto de los jóvenes desmovilizados implicaría pensar en la oportunidad que tienen de ser nuevamente miembros de una sociedad y de establecer pretensiones socialmente aceptadas. En este aspecto, Honneth (1997) menciona: "tener derechos significa poder establecer pretensiones socialmente aceptadas, esto dota al sujeto singular de la oportunidad de una actividad legítima, en conexión con la cual él puede adquirir conciencia de que goza del respeto de los demás. El carácter público de los derechos es aquello por lo que ellos autorizan a su portador a una acción perceptible por sus compañeros de interacción, lo que les concede la fuerza de posibilitar la formación del autorrespeto; pues con la actividad facultativa de la reclamación de derechos al singular se le da un medio simbólico de expresión cuya efectividad social siempre le puede demostrar que encuentra reconocimiento general en tanto que persona moralmente responsable" (p. 147). 
En el escenario normativo del reconocimiento, toda persona es tenida en cuenta como portadora de derechos cuando se le reconoce como actor social. Los jóvenes, incorporados a su derecho a la educación, recuperan y ejercen, en el marco de las relaciones, su condición de sujetos políticos y sociales. De esta manera, la escuela se concibe como un escenario propicio para la socialización de los actores implicados en la formación. En efecto, nos estamos refiriendo a un lugar que propicia una actividad de socialización permanente, desplegada como se ha dicho, en la dialéctica de la reflexividad y de la alteridad en la figura del "reconocimiento mutuo".

De lo anterior se desprende que los jóvenes desmovilizados son sujetos morales que desean reorientar su vida desde unas relaciones de convivencia que tiendan a la vida buena con y para otros en el contexto de instituciones de justicia. Así pues, la escuela con ayuda de los orientadores escolares necesita brindar espacios que promuevan en los sujetos la importancia de reconstruir trayectorias de vida establecidas en la justicia, la estima de sí y el respeto de sí mediante una intencionalidad ética que establezca una vida buena para sí y para el otro. Ricœur (2003) llama intencionalidad ética "a la intencionalidad de la buena vida con y para otro en instituciones justas" (p. 176). Este principio, que tiene como referencia la estima de sí en el plano de lo teleológico y el respeto de sí en el escenario deontológico en instituciones de justicia, debe estar aplicado a la acción y al reconocimiento del otro.

Un aspecto que contribuye a la formación ético-política del joven está en el carácter de su propia estima al reconocerse como agente que, en la medida en que aprecia sus acciones, es principio de la acción. Así, el orientador escolar necesita promover en el agente educativo la idea de que él está llamado a realizar acciones que van a reorientar su vida para alcanzar una vida buena. En efecto, muchos de los jóvenes desmovilizados que han asumido experiencias de guerra y que se reincorporan al contexto escolar desean vivir de otra manera, "son personas con deseos de vivir, de querer ver cara a cara el mundo, con expectativas de un mundo mejor", decía una orientadora (E). Ahora bien, el sujeto que llega a la escuela, en la medida en que se ha propuesto un fin, examina el modo y el medio de alcanzarlo: en este caso, el juicio de deliberación es importante para la elección del medio más adecuado que le permita alcanzar una vida buena que tenga como pregunta orientadora: ¿qué debo hacer?

La pregunta por la vida buena en Aristóteles (2003) constituye el punto referencial entre el concepto ético de Paul Ricœur y Jürgen Habermas (Montoya-Londoño, 2010). Para Ricœur (2003), el primer componente del objetivo ético es lo que Aristóteles llama "vivir bien", "vida buena". Para Jürgen Habermas (1991), sea el problema de que se trate, en lo que corresponde al vivir bien, la pregunta ¿qué debo hacer? cobra un significado pragmático, ético o moral en los fines del sujeto. Así, se espera en cada caso de la razón práctica operaciones y resultados distintos según sea la aplicación. Se trata de la justificación de decisiones entre posibilidades alternativas de acción. En efecto, formar para el juicio ético, moral y pragmático de los jóvenes que llegan a la escuela, es relevante para que todas sus acciones estén involucradas en lo que corresponde a la intencionalidad ética del vivir bien. La estructura de las relaciones del reconocimiento social debe contribuir a la reconstrucción de una sociedad largamente fracturada por el conflicto armado en Colombia. Sin embargo, para que se consolide el proyecto de una sociedad democrática, equitativa, pacífica y sostenible, la escuela necesita tener presente en los procesos de acogida de los jóvenes desmovilizados el deber de la memoria y la justicia. 
Sobre la memoria y la justicia de los jóvenes desmovilizados

en el proceso de la orientación escolar

En nuestro recorrido, nos ocuparemos ahora de comprender el papel de la memoria y la justicia en el reconocimiento de jóvenes desmovilizados de las organizaciones implicadas en el conflicto armado en Colombia. En este apartado, destacamos el valor formativo de la experiencia y la narración en el proceso de vinculación de los jóvenes en el sistema educativo. Queremos insistir en que la escuela como agente reconstructor requiere tener presente que la vida de los jóvenes que participaron en la guerra, está mediada por una historia de violencia, caracterizada por las manifestaciones más extremas de barbarie, que vulneró su dignidad como personas. En este panorama, encontramos que algunos de los jóvenes desmovilizados son víctimas del conflicto; puesto que nacieron allí o fueron reclutados indiscriminadamente o ingresaron a las filas, porque no tuvieron alternativas económicas, sociales y políticas. Han estado expuestos a múltiples violencias basadas en relaciones de abandono, empobrecimiento, injusticia, guerra, pobreza, inequidad. Dicho de otro modo, han sido jóvenes víctimas de la guerra. Sujetos que al dejar los grupos armados y dar paso a la vida civil, se han hecho visibles porque al reincorporarse actualizan una historia cargada de injusticias que se develan gracias a la memoria.

Por eso, el reconocimiento de los jóvenes desmovilizados del conflicto armado no debería pensarse solamente en el tiempo presente. El sujeto está en capacidad de hacer "conciencia reflexiva de sí mismo" a partir de una historia narrativa de su vida (Ricœur 2004, 2006). La narración como práctica que contribuye a los procesos de reconocimiento en la escuela, debe convertirse en una actividad liberadora y en una producción indefinida de sentidos que promueva en el joven desmovilizado la oportunidad de contar la propia vida. Para los jóvenes, reconocerse a sí mismo es el espacio para tomar conciencia de ciertas realizaciones que le permitirán reconfigurar su proyecto de vida. Es una actividad pedagógica que tiene poder develatorio y reparador.

En lo que respecta al tiempo y a la constitución narrativa de la identidad personal del sujeto desmovilizado, conviene recordar aquí a Manuel Reyes Mate (2006), que dijo que "la historicidad es una construcción del presente que busca en el pasado razones para una estrategia de la razón actual; el tiempo reconoce al pasado voz propia, capacidad de interpelación independientemente de los intereses del presente" (p. 137). Según esta concepción, se requiere construir un enfoque anamnético de la justicia, de los derechos y del reconocimiento social en los procesos educativos, de tal manera que la escuela pueda destacar las experiencias y las voces de los jóvenes desmovilizados como sujetos hablantes que pueden decir cosas acerca de la experiencia del conflicto. De este modo, "una cultura de la memoria deberá tener en cuenta la posibilidad de las dos lecturas de la misma historia - la de las víctimas y los victimarios - y estar muy atenta al monopolio hermenéutico de la misma" (Mate, 2006, p. 64). Por esta razón, nos atrevemos a afirmar que la memoria de las personas que fueron

9 La escuela en sus procesos académicos y formativos, y la sociedad en general, requieren la importancia de una justicia anamnética que responda a las experiencias de injusticia. Siendo así, ¿qué significa una justicia que tenga en cuenta el pasado? Reyes Mate (2003) señala al respecto: significa, en primer lugar, responder a una sensibilidad moral nueva. Se multiplican las señales que demandan una comprensión de la justicia que desborde los estrechos límites del tiempo y del espacio en la que permanecía encerrada desde sus inicios; en segundo lugar, la justicia anamnética es entender la justicia como respuesta a la experiencia de injusticia; en tercer lugar, a la justicia anamnética pertenece el descubrimiento de que hay dos visiones de la realidad: la de los vencedores y la de los vencidos (pp. 338-345). 
protagonistas, espectadores y víctimas de la violencia no se agota en una exigencia conceptual. Esto es que la experiencia trágica de las personas que han vivido la guerra requiere una justificación social mientras que el trabajo de la memoria es recordar para comprender que la violencia pasada ha tenido unas consecuencias políticas, sociales y morales que están presentes; que el reconocimiento de esos daños sociales y políticos afecta a la legitimidad de nuestro presente, construido sobre las espaldas de muchas personas (Mate, 2003).

Estamos conscientes de que nuestro presente está construido sobre las injusticias pasadas y nosotros, los presentes, somos herederos de ese pasado injusto. Por esta razón, para Manuel Reyes Mate (2003), "la memoria pretende actualizar la conciencia de una injusticia pasada, mientras que el olvido la cancela, con lo que se hace cómplice de la injusticia" (p. 117). Este es el punto: memoria es denuncia de la injusticia y olvido es sanción de la injusticia. De lo dicho, el recuerdo mantiene vivos, vigentes, los derechos que una vez les fueron negados o pisoteados a las personas. Es cierto: la memoria no es un "adorno", sino un acto de justicia. Y si la memoria es un acto de justicia, entonces no podemos frustrar a las víctimas, en este caso a los jóvenes, ofreciéndoles, por ejemplo, una justicia retórica. Lo que está en juego, no es solo el reconocimiento del derecho a la felicidad de las personas víctimas del conflicto, sino mucho más: la exigencia de felicidad, de esa felicidad que tuvieron tantos seres humanos y de la que a ella se les privó injustamente (Mate, 2003).

Si la memoria es un acto de justicia, los orientadores escolares pueden valerse de la narrativa, por ejemplo, como práctica generadora de reconocimiento que promueva en los jóvenes el intercambio de experiencias como acto de liberación y como comprensión de sí. El recurso de la narrativa es imprescindible en los procesos de atención a los jóvenes desmovilizados: "ellos llegaban acá y te podían hablar una hora. Y yo decía en esa hora, Dios mío, qué les voy a decir a todo lo que me están contando y ellos hablaban, hablaban, mientras que yo de una u otra forma daba algún apoyo, pero una cosa mínima y ellos se paraban y me decían: Profe, muchísimas gracias, eso era lo que necesitaba, y yo decía pero yo no les dije nada. Entonces era como permitirles hacer esa catarsis y ya", narra una orientadora escolar (E). En efecto, el joven posee la singularidad personal asociada al poder narrar y narrarse desde una posición que mira hacia el pasado y hacia el futuro. Así pues, la palabra se convierte como mediación pedagógica en la forma reflexiva del contarse; una mediación que favorece en el sujeto por medio de la memoria y la promesa el reconocimiento presente de su propia existencia; una forma que propicia que en los sujetos se despliegue el compromiso explícito con la memoria y la justicia. Desde esta perspectiva, la problemática del reconocimiento de sí debe alcanzar simultáneamente dos cimas con la memoria y la promesa. La primera para mirar hacia el pasado; la segunda, para mirar hacia el futuro (Ricœur, 2004, 2006).

El orientador escolar como gestor de prácticas de reconocimiento necesita ir generando una pedagogía social de la memoria que promueva el papel transformador que poseen los jóvenes desmovilizados. Esta tarea de reconstrucción de la memoria se emprende reconociendo la nueva visibilidad de los jóvenes en el fin del conflicto armado. Una visibilidad marcada por la heterogeneidad de relatos, significados, experiencias y lenguajes que se registran en el horizonte de la barbarie y de la violencia proveniente del conflicto armado en Colombia. Sin embargo, la escuela requiere trabajar además con los que no son o no han sido víctimas directas para promover acciones colectivas orientadas a transformar las prácticas de violencia, para hacer de la convivencia pacífica un gran acuerdo político y cultural en los diversos territorios.

Los orientadores escolares se ven obligados a indagar en estrategias de recuperación de la memoria. "Rescatar la memoria histórica de los jóvenes que llegan a la escuela es primordial para su atención y desarrollo", decía uno de los orientadores (E). Por eso, la escuela requiere promover estrategias que destacan el valor formativo y expresivo que tienen las producciones documentales y culturales; las prácticas artísticas como murales, canciones, pinturas, obras de teatro; las prácticas sociales como galerías, monumentos, celebraciones religiosas. Además, las estrategias que dignifican la memoria, como hecho reparador, privilegian acciones como: a) facilitar la elaboración del duelo; b) incorporar la experiencia, los sentimientos y llenar de sentido a lo que ya pasó; c) resignificar el cuerpo y activar procesos de liberación y sanación; d) evocar los recuerdos que transitan por el olvido. Sin apartarnos del sentido reparador de la memoria, conviene recordar aquí a Mario Benedetti (2001). Para el poeta, como para nosotros, el olvido está lleno de memoria. Una memoria repleta de fantasmas que peregrinan por el olvido. Sin embargo, "el día o la noche en que el olvido estalle salte en pedazos o crepite/los recuerdos atroces y los de maravilla quebrarán los barrotes del fuego arrastrarán por fin la verdad por el mundo y esa verdad será que no hay olvido" (p. 13).

\section{Consideraciones finales}

Consideramos que los modelos de reconocimiento social aportan a los orientadores escolares de las instituciones educativas, en particular en la educación básica y media, referentes conceptuales que contribuyan a la comprensión de los procesos de inclusión a la escuela de jóvenes desmovilizados 
del conflicto armado. Esto es, que los orientadores escolares requieren comprender la realidad del sujeto desmovilizado mediante prácticas que partan del reconocimiento.

En efecto, interpretar la pretensión de los jóvenes por un reconocimiento intersubjetivo debe poner en primer lugar, la progresión de la subjetividad, es decir, sobre cómo el conocimiento de sí ha de buscarse siempre en la relación consigo mismo. Luego, la de la alteridad, en el sentido de un reconocimiento recíproco que base la comprensión del otro por medio de lazos afectivos entre un número restringido de personas. Y, finalmente, en un tercer plano más desarrollado, la importancia del rasgo de ser reconocido en un escenario normativo que involucre el despliegue social de un reconocimiento mutuo. Así, la relación consigo mismo se nutre de la relación con el otro: no hay yo sin el otro.

La escuela como territorio de paz en un escenario de posconflicto armado requiere ser un lugar que promueva los diferentes estadios de la sociabilidad humana. En la medida en que la escuela se involucre en los procesos de protección y acogida de los jóvenes que poco a poco se han ido desmovilizando del conflicto armado, el reto de ampliar el concepto de política de reconciliación e incluirlo estructuralmente en la agenda de democratización que fomente la inclusión social, la equidad económica, el desarrollo humano integral y sostenible, la participación de los jóvenes en el poder político y, en conjunto, el respeto pleno de los derechos humanos, será posible. Esto implica reconstituir espacios de sociabilidad y de vida política que permitan desde la educación, la consolidación de un proyecto de reconstrucción de ciudadanía y de la promoción de una cultura de paz. Para esto, la escuela está llamada a ofrecer prácticas generadoras de reconocimiento que aporten a la vida de los jóvenes que participaron en los grupos alzados en armas, oportunidades que les dejen reorientar su trayectoria de vida con dignidad y justicia.

Por otro lado, las tres formas de reconocimiento del amor, del derecho y la valoración social, propuestos por Honneth, constituyen las condiciones sociales bajo las cuales los sujetos pueden llegar a una posición positiva frente a sí mismos, debido a la adquisición progresiva de autoconfianza, autorrespeto y autoestima. De acuerdo con lo anterior, se comprendió que el primer aspecto que se requiere es el camino que tiene el joven en lo que corresponde al descubrimiento de sus emociones. Debido a esta perspectiva de los sujetos, hay una articulación que permite "pasar de la tristeza, que implica conocer las formas de destrucción de la vida de jóvenes, niñas y niños, a la esperanza, que es el horizonte de la paz" (Alvarado, Ospina, Quintero, Luna, Ospina \& Patiño, 2012, p. 10). En este sentido, vimos que las emociones proporcionan razones para explicar las acciones humanas en general, y la vida moral en especial de los jóvenes que asumen el reto de reintegrarse a la vida civil. Por eso, en lo que respecta a la dimensión del reconocimiento afectivo, las emociones constituyen elementos esenciales para el potencial desarrollo de la confianza en sí mismos de los jóvenes que hicieron parte del conflicto armado en Colombia. Así, la intervención ética y política de la pedagogía requiere seguir incentivando en los jóvenes una formación en el "autocuidado" que tenga como objetivo la transformación de "sí mismo", del lazo que puede y debe establecer con los otros, y del desarrollo de emociones y sentimientos que propicien en los sujetos el cultivo de unas relaciones de convivencia justas y pacíficas.

Sin embargo, la esfera de las emociones que propicie una fenomenología de sí, no es suficiente para la comprensión de un reconocimiento que se ocupe de la dimensión social. En este caso, se describió que el joven desmovilizado no puede llegar al entendimiento de sí mismo como 
portador de derechos, si no posee un saber acerca de qué obligaciones normativas requiere cumplir frente a los otros. Desde esta perspectiva, en el marco normativo, el joven desmovilizado se constituye como un sujeto de derechos y la escuela se convierte como un medio y un fin para su reinserción y reparación. De este modo, los jóvenes incorporados a su derecho a la vida escolar asumen relaciones político-sociales.

No obstante, para que se desarrolle una valoración plena de las realizaciones individuales y colectivas de los jóvenes desmovilizados, el reconocimiento de sus derechos, deberes y capacidades en clave éticopolítica, la escuela debe tener presente la memoria, el tiempo y la justicia que subyacen en el devenir de estos jóvenes. Siendo así, el último recorrido de este trabajo comprendió la idea según la cual la memoria es importante para elaborar una teoría de la justicia que proteja y proyecte a la escuela como territorio para la convivencia pacífica. Los desafíos de la escuela en lo que respecta al reconocimiento de los jóvenes desmovilizados con historias emocionales diversas, que requieren construir nuevas formas de ciudadanía para tejer sentidos de pertenencia, tienen qué ver con el afianzamiento de los vínculos afectivos, jurídicos y sociales. Por eso, la escuela — con ayuda del orientador escolar - está llamada por medio de prácticas narrativas a formar seres capaces de reconocer su identidad como un proceso en permanente reconstrucción, que abre posibilidades para reinventarse constantemente en virtud de trascender la frontera del individualismo y anclar sus historias de vida con otras personas.

La escuela y la sociedad en general necesitan formar para la pluralidad y el reconocimiento en el papel activo del sujeto desmovilizado que construye relaciones con el otro. Esto implica siempre la participación no solo de los jóvenes desmovilizados, sino de la comunidad en general. Es necesario resaltar la importancia del reconocimiento en el papel activo del sujeto, en la medida en que es él quien ejecuta la acción de reconocer algo fuera de su mismidad (Ricœur, 2006); dicha acción de reconocimiento, aún envuelta en su carácter subjetivo, muestra también una diferencia notable en la medida en que la subjetividad y la mismidad se constituyen en su relación con el otro, con el encuentro del ente distinto y opuesto. De este modo, propiciar posiciones éticas, políticas y jurídicas que favorezcan el reconocimiento para superar la asimetría entre el yo y el otro se convertirá en condición fundamental para la construcción de una sociedad más justa y pacífica.

Retomando la tesis central de esta disertación, podemos concluir diciendo que el potencial emocional, jurídico y social de las prácticas generadoras de reconocimiento en el contexto escolar, facilitará a los orientadores escolares la atención de jóvenes que vivieron en contextos de guerra con un criterio ético y político basado en la acogida y la protección en las dinámicas de inclusión; en el respeto de los derechos; en la solidaridad desde la estima de sí y del otro; en el perdón, la memoria, la reparación y las garantías de no repetición. Las prácticas de reconocimiento y de orientación se constituirán en experiencias significativas en las trayectorias vitales de los jóvenes. Finalmente, hacer de la escuela un espacio de construcción social para el reconocimiento de jóvenes desmovilizados de los grupos armados en Colombia, se deberá más que a la necesidad de enfrentar la coyuntura que vivimos, a la justificación que tenemos de saber quiénes somos.

\section{Sobre los autores}

Henry Camilo Bejarano-Sanabria es profesional en filosofía y letras, Universidad de La Salle. Magíster en educación, Pontificia Universidad Javeriana. Director de investigación de la Corporación de Educación Tecnológica Colsubsidio-Airbus Group, CET.

Ricardo Mauricio Delgado-Salazar es doctor en ciencias sociales, niñez y juventud, Universidad de Manizales-CINDE. Profesor titular de la Facultad de Educación, Pontificia Universidad Javeriana, Bogotá. Coordinador del grupo de investigación Educación para el Conocimiento Social y Político.

\section{Referencias}

Alvarado, Sara Victoria; Ospina, Héctor Fabio; Quintero, Marieta; Luna, María Teresa; Ospina, María Camila \& Patiño, Jhoana A. (2012). Las escuelas como territorios de paz. Construcción social del niño y la niña como sujetos políticos en contextos de conflicto armado. Luis Tapia \& María Isabel Orofino, prólogo. Ciudad Autónoma de Buenos Aires: CLACSO, Universidad de Manizales, CINDES, 2012. Disponible en: http://biblioteca.clac so.edu.ar/clacso/posgrados/20120910105957/ LasEscuelascomoTerritoriosdePaz.pdf

Aristóteles (2003). Ética a Nicómaco. Julio Pallí-Bonet (trad.), Madrid: Gredos.

Benedetti, Mario (2001). El olvido está lleno de memoria. Madrid: Visor Libros, Colección Visor de Poesía.

Habermas, Jürgen (1991, 2000). Del uso pragmático, ético y moral de la razón práctica. En Aclaraciones a la ética del discurso. Manuel JiménezRedondo (trad.), Madrid: Trotta. Disponible en: http://www.inau.gub.uy/biblioteca/seminario/ eticadiscurso.pdf

Hegel, Georg Wilhelm Friedrich (1973). Fenomenología del espíritu. México: Fondo de Cultura Económica, FCE. 
Hegel, Georg Wilhelm Friedrich (2006). El Sistema de la eticidad. Buenos Aires: Editorial Quadrata.

Honneth, Axel (1997). La lucha por el reconocimiento. Por una gramática moral de los conflictos sociales. Barcelona: Ed. Crítica.

Honneth, Axel (2009). Crítica del agravio moral. Patologías de la sociedad contemporánea. Peter Storandt-Diller \& Gustavo Leyva, trads., Buenos Aires: Fondo de Cultura Económica, FCE.

Honneth, Axel (2010). Reconocimiento y menosprecio. Sobre la fundamentación normativa de una teoría social. Buenos Aires: Katz.

Mate, Manuel Reyes (2006). Contra lo políticamente correcto. Política, memoria y justicia. Buenos Aires: Editorial Altamira.

Montoya-Londoño, Mauricio (2010). Lo justo: entre lo bueno y lo legal. Un diálogo entre la intencionalidad ética de Paul Ricœur y el constructivismo político de John Rawls. Tesis para optar el título de doctor de Filosofía. Facultad de Filosofía, Pontificia Universidad Javeriana, Bogotá. Disponible en: http://repository.javeriana.edu.co/bitstream/10554/1096/1/ MontoyaLondo\%C3\%B1oMauricio2010.pdf

Nussbaum, Martha C. (2007). Las fronteras de la justicia. Consideraciones sobre la exclusión. Barcelona: Ediciones Paidós Ibérica.

Nussbaum, Martha C. (2008). Paisajes del pensamiento: la inteligencia de las emociones. Madrid: Ediciones Paidós Ibérica.

Nussbaum, Martha C. (2010). Sin fines de lucro. Por qué la democracia necesita de las humanidades. María Victoria Rodil, trad., Buenos Aires, Madrid: Katz Editores.

Ricœur, Paul (2001). Amor y justicia. Madrid: Caparrós Editores.

Ricœur, Paul (2003). Sí mismo como otro. Séptimo estudio: El sí y la intencionalidad ética. Octavo estudio: El sí y la norma moral. Agustín Neira-Calvo (trad.), Madrid: Siglo XXI de España Editores.

Ricœur, Paul (2004). La memoria, la historia, el olvido. Buenos Aires: Fondo de Cultura Económica, FCE.

Ricœur, Paul (2006). Caminos del reconocimiento. Tres estudios. México: Fondo de Cultura Económica, FCE. 